\title{
Emerging Patterns of Neuronal Responses in Supplementary and Primary Motor Areas during Sensorimotor Adaptation
}

\author{
Rony Paz, ${ }^{1,2}$ Chen Natan, ${ }^{1}$ Thomas Boraud, ${ }^{3}$ Hagai Bergman, ${ }^{1,2}$ and Eilon Vaadiaa ${ }^{1,2}$ \\ ${ }^{1}$ Department of Physiology, Hadassah Medical School, and ${ }^{2}$ The Interdisciplinary Center for Neural Computation, The Hebrew University, Jerusalem 91120 , \\ Israel, and ${ }^{3}$ Laboratoire de Neurophysiologie, Centre National de la Recherche Scientifique Unité Mixte de Recherche 5543, Université Victor Segalen, 33076 \\ Bordeaux, France
}

Acquisition and retention of sensorimotor skills have been extensively investigated psychophysically, but little is known about the underlying neuronal mechanisms. Here we examine the evolution of neural activity associated with adaptation to new kinematic tasks in two cortical areas: the caudal supplementary motor area (SMA proper), and the primary motor cortex (MI). We investigate the hypothesis that adaptation starts at premotor areas, i.e., higher in the hierarchy of computation, until a stable representation is formed in primary areas. In accordance with previous studies, we found that adaptation can be characterized by two phases: an early phase that is accompanied by fast and substantial reduction of errors, followed by a late phase with slower and more moderate improvements in behavior. We used unsupervised clustering to separate the activity of the single cells into groups of cells with similar response patterns, under the assumption that each such subpopulation forms a functional unit. We specifically observed the number of clusters in each cortical area during early and late phases of the adaptation and found that the number of clusters is higher in the SMA during early phases of adaptation. In contrast, a higher number of clusters was observed in MI only during late phases. Our results suggest a new approach to analyze responses of large populations of neurons and use it to show a hierarchy of dynamic reorganization of functional groups during adaptation.

Key words: motor learning; visuomotor rotations; unsupervised clustering; motor cortex; plasticity; extracellular; monkeys

\section{Introduction}

Motor adaptation and skill learning is characterized by an early rapid phase that induces substantial improvement in behavior, followed by a slower phase with more moderate improvements (Karni et al., 1998). In several studies, the late phase, but not the early, was accompanied by changes in primary motor cortex (MI): imaging techniques revealed increased activity when humans learned new sequential finger movements (Ungerleider et al., 2002), and motor map reorganization and synapse formation have been observed when rats learned a novel multijoint reachand-grasp task (Kleim et al., 2004). These findings, together with a diversity of findings showing learning-dependant plasticity in MI (Sanes and Donoghue, 2000), suggest that a representation of the newly acquired skill is formed in MI during the late stages of learning, hence allowing for efficient generation and control of well trained movements (Porter and Lemon, 1993; Scott, 2004). Lately, several studies have reported changes in MI within a time scale of one session, i.e., dozens of trials (Laubach et al., 2000; Li et al., 2001; Cohen and Nicolelis, 2004). We recently

Received Sept. 28, 2004; revised Sept. 13, 2005; accepted 0ct. 16, 2005.

This work was supported in part by Center for Excellence Grant 8006/00 administered by the Israel Science Foundation, by Bundesministerium für Bildung und Forschung/Deutsch-Israelische Projektkooperation, by Grant 2001073 administered by the Binational Science Foundation, and by a special contribution from the Rosetrees Trust. We thank Amir Globerson and Naama Parush for helpful discussions.

Correspondence should be addressed to Rony Paz, Center for Molecular and Behavioral Neuroscience, Rutgers University, 197 University Avenue, Newark, NJ 07102. E-mail: rony@axon.rutgers.edu.

D0I:10.1523/JNEUROSCI.0164-05.2005

Copyright $\odot 2005$ Society for Neuroscience 0270-6474/05/2510941-11\$15.00/0 reported that adaptation to visuomotor rotational transformations induces specific modifications in the activity of MI neurons (Paz et al., 2003). These changes evolved slowly within one session and were prominent only when behavior reached a plateau.

The late occurrence of learning-related changes in MI suggests that initial learning was mediated via other areas (Doyon et al., 1997; Hikosaka et al., 2002; Ungerleider et al., 2002). One candidate for mediating learning and adaptation is the caudal supplementary motor area (SMA proper), a premotor cortical area that plays a major role in the planning and generation of simple and complex movements (Tanji, 1994). It has direct corticospinal projections (He et al., 1993, 1995) and interconnects with other premotor areas (Luppino et al., 1993), and its activity is tightly linked to the preparation for and execution of movement (Alexander and Crutcher, 1990; Crutcher and Alexander, 1990; PadoaSchioppa et al., 2004). Recently, the SMA was shown to participate in learning of new sensorimotor skills (Nakamura et al., 1998; Padoa-Schioppa et al., 2002; Lee and Quessy, 2003).

Neuronal responses are traditionally analyzed by comparing the evoked activity after a certain behavioral event with the activity before this event, e.g., presentation of a sensory stimulus, initiation of movement. Many times, observing perievent time histograms (PETHs) in a certain time window and identifying different parameters of the response such as onset, duration, etc., serve to classify neurons according to indices such as phasic versus tonic, late versus early, and increase versus decrease. Moreover, neurons are screened for additional analysis based on their 
responses as regards these criteria. This may result in failure to identify distinct populations of neurons with specific and interesting relationships to behavior. At the other extreme, advanced methods that examine detailed temporal relationships (Rieke et al., 1997) may lose the original shape of the PETHs.

Here we use unsupervised clustering of PETHs, hence obtaining an unbiased classification of neuronal responses and delineating subpopulations of neurons with similar response shapes. We then compared between responses in SMA and MI, before, during, and after adaptation.

\section{Materials and Methods}

The experimental setup, behavioral paradigm, and detailed behavior were the same as described in previous studies (Paz et al., 2003, 2005). Animal care and surgical procedures complied with the National Institutes of Health Guide for the Care and Use of Laboratory Animals (revised 1996) and with the Hebrew University guidelines supervised by the Institutional Committee for Animal Care and Use.

Experimental design. Two rhesus (Macaca mulatta, $\sim 4.5 \mathrm{~kg}$ ) monkeys were trained to move a low-friction manipulandum to control the movements of a cursor on a video screen located $50 \mathrm{~cm}$ from their torso and eyes. The manipulandum moved the cursor from the starting point at the center of the screen (origin) to a visual target in a delayed go-signal paradigm. This required the monkeys to hold (as verified by hand velocity and EMG) the cursor in the origin circle for a random 750-1500 $\mathrm{ms}$ after target onset. The disappearance of the origin was the go signal (Fig. 1a). In each session (day), three consecutive periods took place (Fig. 1b): (1) the preadaptation period, a standard, eight-target task in which the target direction was randomly chosen from eight possible directions uniformly distributed over the circle; (2) the adaptation period, a transformed, one-target task in which only one target (upwards, $90^{\circ}$ ) was presented and a rotational transformation was introduced between the cursor on the screen and the manipulandum; and (3) the postadaptation period, in which the default, eight-target task was presented again. Rotations were $90,45,-45$, or $-90^{\circ}$ and were chosen randomly for each session but fixed for the duration of the adaptation period in a session.

Physiological procedures. The monkeys were implanted with recording chambers $(27 \times 27 \mathrm{~mm})$ above both the right and left hemispheres. Each day, the monkeys were seated in a dark chamber, and eight glass-coated tungsten microelectrodes (impedance, $0.2-1 \mathrm{M} \Omega$ at $1 \mathrm{kHz}$ ) were inserted into each hemisphere. The signals were amplified, filtered, and sorted (MCP-PLUS; Alpha-Omega, Nazareth, Israel), and all spike shapes were sampled at $24 \mathrm{kHz}$. We used a template-based method for real-time isolation of spike shapes (MSD; Alpha-Omega).

Penetration locations and angles were verified by magnetic resonance imaging (Biospec 4.7 Tesla; Bruker BioSpin, Ettlingen, Germany) before and after recordings; 14 coronal slices, $2 \mathrm{~mm}$ wide, were made to help locate the stereotactic coordinates of the central and arcuate sulci. Penetrations into the SMA proper were angled at $30^{\circ}$ to the sagittal plane and advanced from the point of insertion until they reached the medial cor-

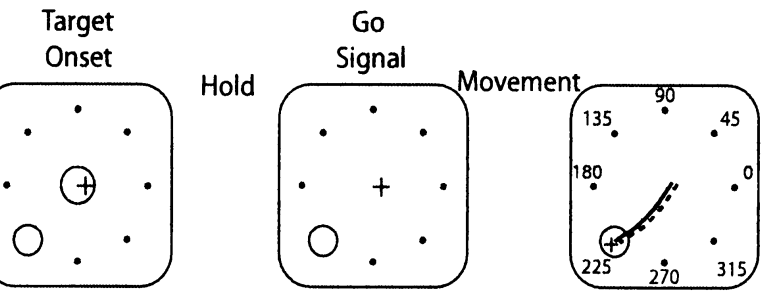

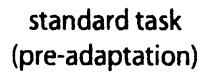

visuomotor rotational transformation (adaptation)

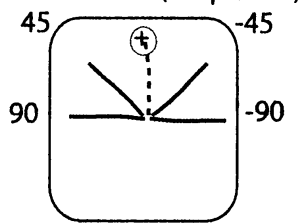

$-90$

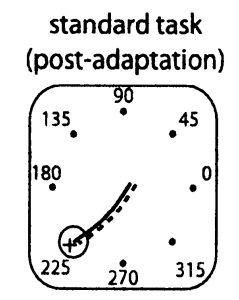

Hand movement — Cursor movement ....

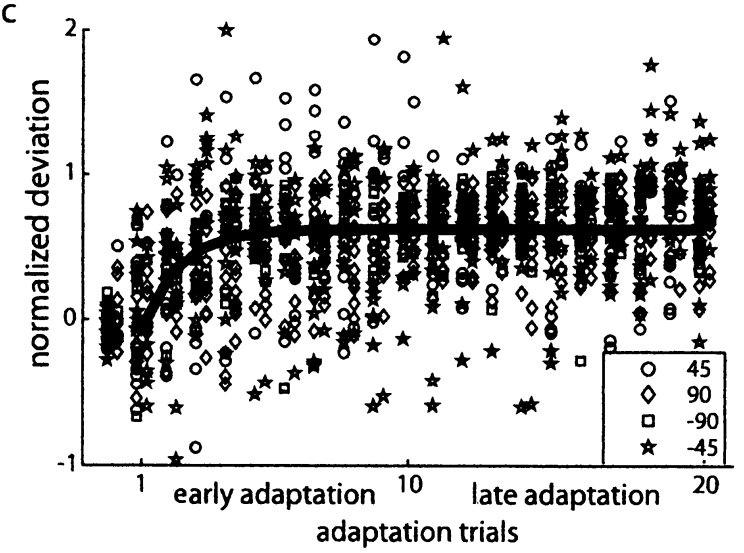

d

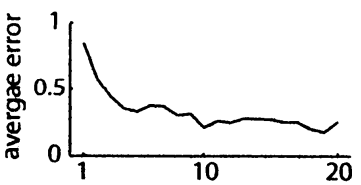

e

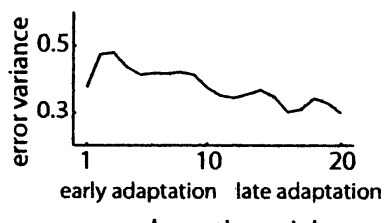

adaptation trials
Figure 1. Experimental design and behavior. $\boldsymbol{a}$, Trial flow, Left, The monkeys had to hold the cursor in the central circle (origin) the hand was in 1:1 relation to the movement of the cursor. Middle, A rotational transformation was introduced, so that movement of the cursor was rotated by $-90,-45,45$, or $90^{\circ}$ to the movement of the hand. Only one of these four transformaby the transformation imposed in the session. Data are pooled from all sessions and four different transforms performed by both Average error, One minus the average (over sessions) of the normalized deviations presented in c. $\boldsymbol{e}$, Error variance is the variance (over sessions) of the normalized deviations presented in $\boldsymbol{c}$.

tex. Generally, this meant that the electrodes were advanced through cortex into white matter and through that white matter before reaching SMA. The pattern "units-white matter-units" was used as an indicator of recording location. At the end of each session, we examined the activity of neurons evoked by passive manipulation of the limbs and applied intracortical microstimulation (ICMS) with trains of $200 \mu$ s cathodal pulses at $300 \mathrm{~Hz}$ with an intensity of $10-80 \mu \mathrm{A}$. Typical train durations were $50 \mathrm{~ms}$ for MI and $100 \mathrm{~ms}$ for SMA. For MI, we examined penetrations in which ICMS evoked arm movements around one joint with an intensity of $\leq 40 \mu \mathrm{A}$.

Data analysis. A "normalized deviation" was calculated for each movement: the direction (angle) of the visual target was subtracted from the direction of the actual hand movement (taken at peak velocity), and the result was divided by the rotational transformation imposed in the session. Thus, when the movement is in the direction of the visual target, this measure is close to zero, but, as adaptation progresses and the discrepancy is closer to the required transformation, it approaches one. Note that the measure uses both the sign and the amplitude of the transformation and therefore reflects both aspects. "Average error" is taken to 
be one minus the average over sessions of the normalized deviation for each movement in the adaptation period, and "error variance" is taken as the variance over sessions.

For each cell, activity was calculated for two different epochs of a trial. The first is termed "preparatory activity" (PA) because it covers activity from 0 to $500 \mathrm{~ms}$ after the target onset, i.e., activity during a hold period. The second is "movement-related activity"(MRA), so termed because it was measured from -200 to +300 ms around initiation of movement, an epoch that contains most of the beginning of muscle activity and neuronal responses that generate the movement but contains fewer feedback effects and sensory-driven movement corrections. Thus, it contains the motor command that initializes the movement. Movement initiation was calculated off-line by an onset algorithm based on the velocity profile.

Spikes were recorded with $1 \mathrm{~ms}$ precision and counted in $10 \mathrm{~ms}$ bins for each trial, averaged for each bin across all trials from a behavioral period (always $\geq 10$ trials), smoothed using a Gaussian window (with variance equal to $30 \mathrm{~ms}$ ), and $z$-score transformed to form the PETH. Each PETH is therefore a vector of length 50 (500 ms in $10 \mathrm{~ms}$ bins); $x_{i j}$ denotes the activity of cell $i$ at time bin $j$.

For clustering, we used the $k$-means algorithm (Hartigan, 1975), which uses an iterative procedure to minimize the total of within-cluster sums of distances from centroids:

$$
E=\sum_{j=1}^{k} \sum_{i \in C j} d\left(x_{i}, \bar{x}_{j}\right)
$$

where $\bar{x}_{j}$ is the center of cluster $C_{j}$ (the average vector of all cells associated with the cluster), $k$ is the number of clusters, and $d$ is a distance measure traditionally taken to be the Euclidean distance:

$$
d\left(x_{1}, x_{2}\right)=\sqrt{\sum_{j=1}^{50}\left(x_{1 j}-x_{2 j}\right)^{2}} .
$$

To avoid and minimize convergence to local minima, each $k$-means procedure was repeated 50 times, each with a different set of randomly generated centroids, and the run that produced the smallest error was chosen.

Whereas many clustering procedures use $k$-means, different approaches apply different embedding of the original data points (i.e., a representation of the data to be used as input to the clustering algorithm), and this is of high importance to the success of the complete process. We tested four different approaches of embedding space for the PETHs.

(1) "Activity matrix $x$ " is composed of PETHs from different cells of the same condition. Thus, each row is a PETH of a cell, a vector of firing rates, where $x_{i j}$ is the activity of cell $i$ at time bin $j$. In other words, no additional embedding is performed and the original data points are used.

(2) In the "similarity (dissimilarity/affinity) matrix $S$," we followed and used the procedure as described by Schreiber et al. (2003) and Fellous et al. (2004). Briefly, each row represents the vector of similarities between a PETH of one cell and PETHs of all other cells. This is therefore a symmetric matrix with a size of $N \times N$, where $N$ is the number of cells in the sample. Similarity was measured by taking the normalized dot product between pairs of PETHs (i.e., the cosine between the two activity vectors):

$$
S_{i j}=\frac{\vec{x}_{i} \times \vec{x}_{j}}{\left\|\vec{x}_{i}\right\| \times\left\|\vec{x}_{j}\right\|} .
$$

(3) The "principal component analysis (PCA) matrix $p$ " was performed separately for each case (cortical area + activity epoch + behavioral period) (Fig. 2). The eigenvectors of the covariance matrix were sorted according to their eigenvalues (explained variance), and the principal components were calculated (the dot product between a data vector and the eigenvectors, i.e., the projection of a data point on the eigenvectors). Each cell was then represented by its $J$ first principal components, i.e., the components corresponding to the $J$ eigenvectors with the highest explanatory power; hence, $p_{i j}$ is the component $j$ for cell $i$. Because PCA was used only for clustering purposes and is not the focus of this study, we only further investigated its relationship to the quality of clustering. We therefore tested the use of $2,3,4,6,8,10$, and 20 coefficients for clustering, observed number of clusters ( $k$ values) for each number of coefficients, and verified that examples of the obtained clusters were also similar in shape for different numbers of coefficients.

(4) In "spectral clustering," spectral methods use selected eigenvectors of the data similarity matrix to obtain a data representation that can be embedded and clustered in a low-dimensional space. The simplest form of these methods clusters the eigenvectors of the dot-product matrix of the data and is equivalent to clustering the projection of the data points on the eigenvectors of the covariance matrix. In other words, it is similar to the PCA matrix approach discussed above. However, most versions of these methods use Gaussian kernel to create the similarity matrix, and this is equivalent to kernel PCA. Furthermore, some of these methods put emphasis on different normalization procedures that improve the clustering task. We used the algorithm suggested by Ng et al. (2001).

We used two measures to compare approaches and different number of clusters ( $k$ values): "silhouette analysis" (Kaufman and Rousseeuw, 1990) and "gap analysis" (Tibshirani et al., 2001).

Silhouette analysis is a measure of how similar each point is to points in its own cluster compared with points in other clusters. For each point, we calculated $a_{i}=\operatorname{avg}_{j \in C_{k}}\left(d\left(x_{i}, x_{j}\right)\right)$, the average distance (dissimilarity) from all the points in the same cluster. For each point and each cluster that the point does not belong to, we calculated $b_{i k}=\operatorname{avg}_{j \in C_{k}}\left(d\left(x_{i}, x_{j}\right)\right)$, the average distance from all of the points in that cluster, and then took the minimum over all clusters: $b_{i}=\operatorname{Min}_{k}\left(b_{i k}\right)$. The silhouette is defined as

$$
S(i)=\frac{b_{i}-a_{i}}{\operatorname{Max}\left(a_{i}, b_{i}\right)} .
$$

This measure ranges from +1 , indicating points that are very distant from neighboring clusters, through 0 , indicating points that are not distinctly in one cluster or another, to -1 , indicating points that are probably assigned to the wrong cluster. Heuristically, the silhouette measures how well a point is matched to the other points in its own cluster versus how well it would be matched if it was moved to the next closest cluster.

The score for the clustering procedure is the average over all points, i.e.

$$
\bar{S}=\frac{1}{N} \sum_{i=1}^{N} S(i) .
$$

This measure is not monotonous in $k$, the number of clusters.

In gap analysis, $k$-means minimizes the sum of within-cluster distances from centroids $(E)$. However, comparing this across different $k$ values will not do because $E(k)$ is by construction monotone nonincreasing in $k$ (more clusters always means smaller within-cluster distances). An alternative is to look at the relative improvement from $k$ to $k$ +1 , based on the intuition that the relative improvement becomes insignificant when $k$ increases above the "true" $k$, because this is when we start to assign natural groups to separate clusters. To formalize this, gap analysis is based on simulating samples by drawing from a uniform distribution over the space spanned by the real data, calculating $E \_s i m u l a t-$ ed $(k)$ and comparing $\log \left(E \_s i m u l a t e d(k)\right)$ with $\log (E(k))$. The criterion is then as follows (Tibshirani et al., 2001): $K=\arg \min _{k}\{k \mid G(k) \geq G(k+$ $1)-\operatorname{std}(k+1)\}$, where $G(k)$ is the gap curve taken as $\log \left(E \_\right.$simulat$\operatorname{ed}(k))-\log (E(k))$ and $\operatorname{std}(k)$ is the SD of $\log ($ E_simulated $(k))$. We performed 25 simulations to obtain the SDs.

All analyses were performed in Matlab (MathWorks, Natick, MA), with custom software.

\section{Results}

The adaptation period was divided into two stages based on the animals' behavior (Fig. $1 c-e$ ). The initial fast improvement in behavior was considered as the "early period" (trials 1-10), and the slower improvement, which includes a behavioral plateau, was considered as the "late period" (trials 11-20). Trials were therefore divided into four groups: "preadaptation" (during performance of standard movements), "early adaptation," "late adaptation," and "postadaptation," the latter being a second set of 
a

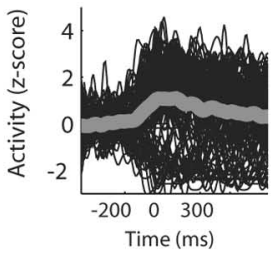

b

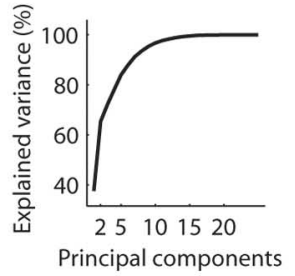

d

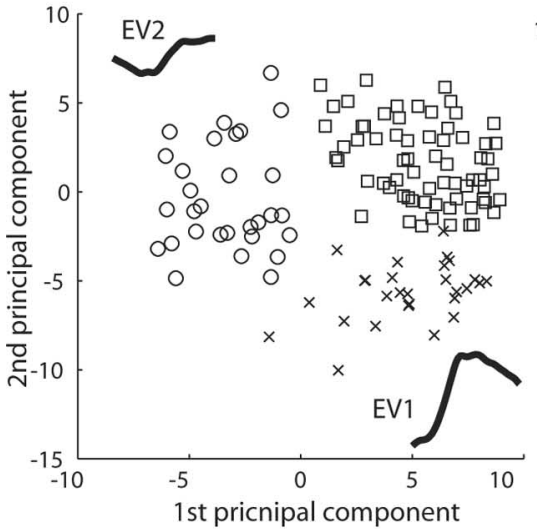

C1

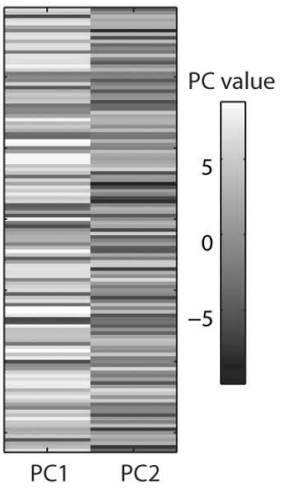

C2

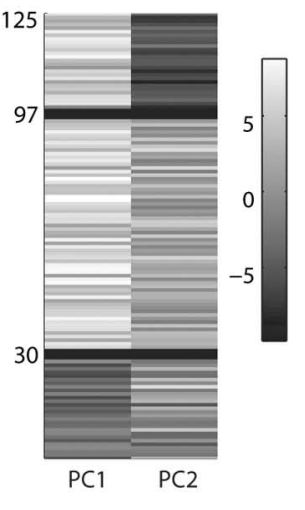

e1

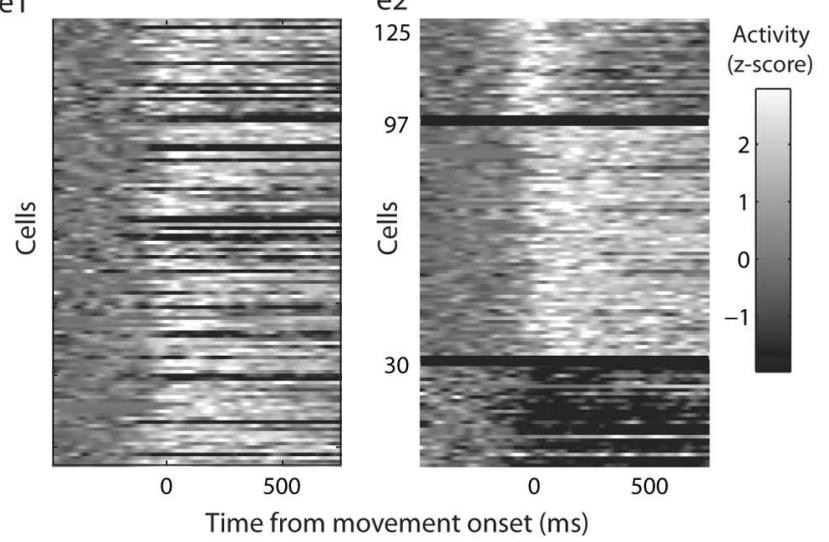

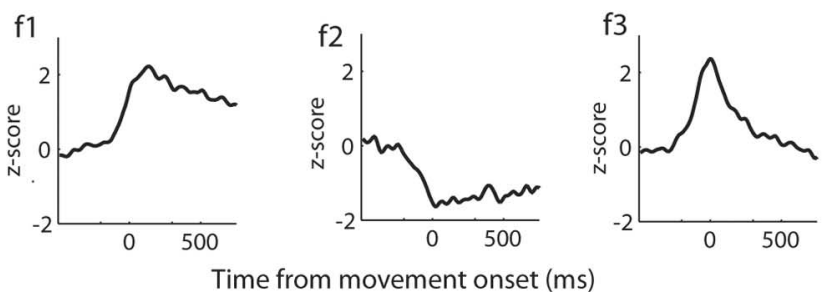

Figure 2. Clustering of neuronal responses. MRA from 125 most responsive (Mann-Whitney test, $p<0.02$ ) cells in Ml during performance of the standard task (preadaptation) is used to demonstrate the clustering procedure. For each cell, spike count was averaged over trials in 10 ms bins, smoothed with a Gaussian window, and $z$-score transformed to produce activity vectors (PETHs, overlaid in $\boldsymbol{a}$, gray thick line is the average of all cells, zero time is the beginning of movement as calculated off-line with a slope algorithm). A subset of $500 \mathrm{~ms}$ (from -200 to 300 , a vector of length 50 ) from each cell was used for the PCA. $\boldsymbol{b}$, Cumulative explained variance as a function of the first (largest) 25 eigenvalues; in general, $<8$ eigenvectors accounted for $>80 \%$ of the variance for all conditions, and this spectrum did not differ significantly across standard trials performed after adaptation. We further differentiated activity involved in planning and preparing for the upcoming movement (PA) from activity that supports the actual generation of movement (MRA). A "case" in this report refers to a combination of cortical area, behavioral period, and epoch of neuronal activity, e.g., PA in MI during early adaptation.

\section{Clustering of neuronal responses}

For each case, we constructed PETHs for all cells that were reliably recorded and fired at least one spike in all behavioral periods. We used $k$-means algorithm for unsupervised clustering of the PETHs of cells within each case and tested four different approaches for embedding the data (see Materials and Methods): (1) using the PETHs themselves; (2) using the similarity matrix between the PETHs; (3) using a more compact representation of the PETHs. For that, we chose PCA to reduce dimensionality, representing each PETH by the first principal components and (4) spectral clustering. In general, all four approaches performed equally well and produced very similar results (indistinguishable centers of the clusters). The PCA approach was slightly superior quantitatively as measured by silhouette analysis, suitable for clustering high-dimensional data and less expensive computationally; we therefore present here the results using this approach. Figure 2 illustrates the clustering procedure for a subset of cells recorded in MI and their MRA. We stress that, although clustering was performed on the principal components, all comparisons were performed on the PETHs, and all figures show the original PETHs. The response pattern or shape of a cluster refers to the PETH averaged across all cells associated with the cluster (i.e., the center of the cluster).

First, we analyzed PA in MI. Figure 3 draws PETHs of all cells as a matrix in which each row represents one cell, and the activity in consecutive time bins ( $x$-axis) is represented in grayscale. During preadaptation (Fig. $3 a$ ), activity consisted of two major active clusters: an early phasic response to the visual cue and a late tonic response. These response patterns are consistent with previous studies (Crammond and Kalaska, 2000) and confirm that, for standard (well trained) movements, the clustering method reproduces ad hoc observations. Also presented are the actual PETHs from 35 cells (Fig. 3a3), superimposed separately for each cluster. For presentation, we took the 35 cells with average distance from the center of the cluster (i.e., we sorted cells according to their distance from the center of the cluster and took the 35 middle ones).

\section{Clusters change during adaptation}

In contrast to the three typical response patterns during preadaptation, clustering of responses during late adaptation revealed a larger number of different subpopulations of cells (Fig. 3b). These subpopulations differed mainly in their temporal profile of activation; more specifically, the separation into more clusters re-

$\leftarrow$

cases. For the illustration here, we use only the first two. c1, Principal components ( $P C$, columns) for all cells (rows) in a random order; the values of the first principal component ( $P C 1$, left column) and the second ( $P C 2$, right column) are represented in grayscale. $d, P C s$ of all cells. The different shapes (x symbols, circles, and squares) correspond to the different clusters formed by $k$-means. Also shown are the shapes of the first two eigenvectors (EV1, EV2). c2, Same as c1, but rows are rearranged according to the association of the cells to the clusters. $\mathbf{e} 1$, PETHs from all cells shown in $\boldsymbol{a}$. Each row is a PETH of one cell, grayscaled by the (z-transformed) firing rate intensity in $10 \mathrm{~ms}$ bins. The rows are randomly ordered. $\mathbf{2} 2$, Same data, but rows are rearranged according to the association of the cells to the clusters. f1-f3, Response patterns, average PETH from all cells within one cluster, for the three clusters separately. 
a1

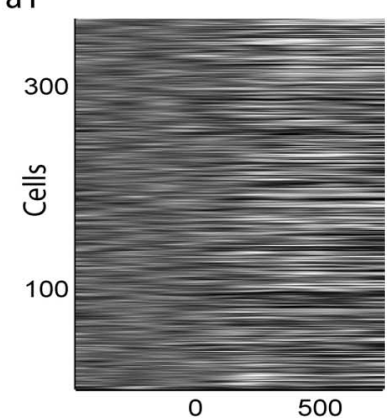

b1

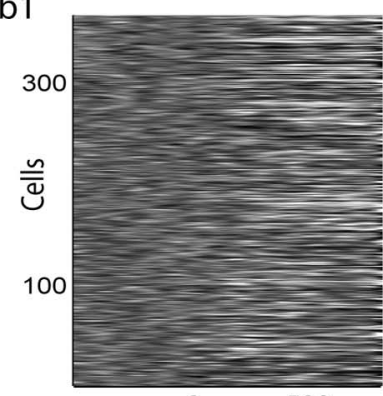

0 z-score $a 2$

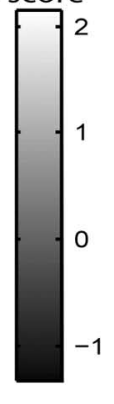

b2
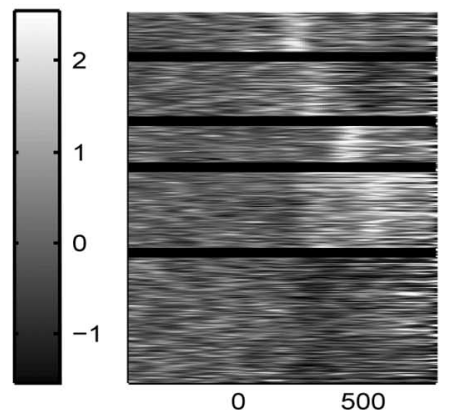

a3

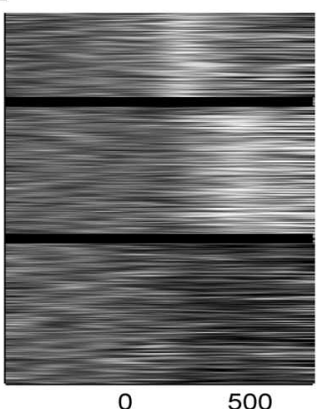

2

(ms)

\section{$\mathrm{C}$}

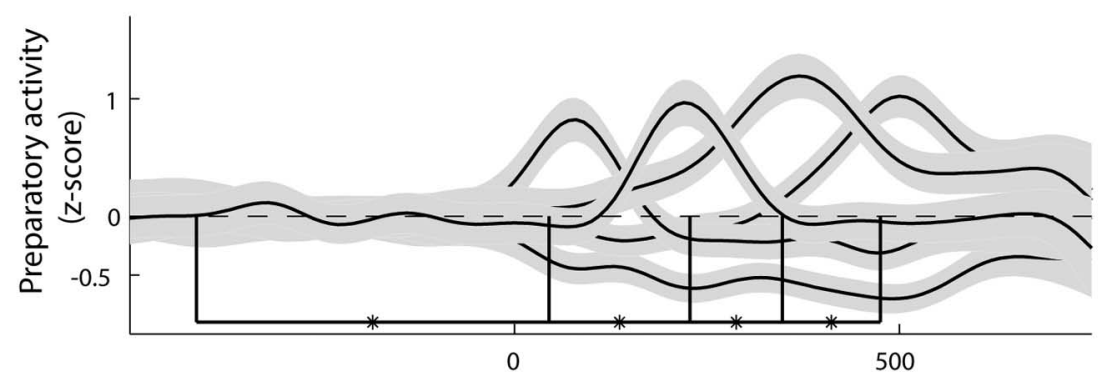

Time from targer appearence (ms)

Figure 3. Clusters during preadaptation versus late-adaptation. $\boldsymbol{a}$, Normalized PETHs of preparatory activity of all neurons in MI of monkey 1 during the preadaptation period (standard task). Each row is a PETH of one cell, grayscaled by the (z-transformed) firing rate intensity in $10 \mathrm{~ms}$ bins. In $\boldsymbol{a} \mathbf{1}$, cells are presented in a random order; in $\boldsymbol{a} \mathbf{2}$, cells are rearranged according to their association to three clusters (borders between clusters are marked in thick black lines). In $\mathbf{a 3}$, PETHs of typical cells are superimposed separately for each cluster; cells within each cluster are sorted according to their distance from the center of the cluster, and the 35 middle ones are taken for presentation. $\boldsymbol{b}$, Same presentation as in $\boldsymbol{a}$ but for preparatory activity in MI during the lateadaptation period. Cells are clustered into five different clusters and reveal different peaks and onsets of activation. c, Response patterns of preparatory activity in MI during late adaptation. Each response pattern is the average PETH (in z-score) from all cells within one cluster, with gray-shaded 0.95 confidence intervals. Vertical lines depict the average peak time of all cells within a cluster (note the close agreement with the peak time of the response pattern), and asterisks represent that the mean peak time was significantly different than the mean peak time of the preceding cluster (one-tailed $t$ test, $p<0.05$ ).

sulted in multiple consecutive phasic peaks of activity (Fig. $3 b 2, b 3)$. This is shown more clearly in Figure $3 c$, in which the shapes of all clusters are overlaid. To test this directly, we calculated the time-to-peak activity for each cell individually and performed various analyses. First, we found that the variance of times-to-peak activity across the whole population of cells in the late-adaptation period was significantly higher than that of cells during preadaptation $(F$ test, $p<0.001)$. In contrast, the variance of the peak amplitudes did not change significantly ( $F$ test, $p>0.1)$. Second, when separated into five different groups corresponding to the different clusters, the means of the groups were significantly different (ANOVA, $p<0.01$ ). Third, when averaged within each cluster (Fig. $3 c$, vertical lines), the means were in close agreement with the peak time of the shapes of the clusters. Finally, sorting the groups according to their mean peak time and comparing each group with the group preceding it revealed that all pairs of clusters differed significantly in their mean peak time [Fig. $3 c$, asterisks (one-tailed $t$ test, $p<0.05$ for all)].

\section{Number of clusters}

To further explore the nature of changes in PETHs during learning, we used two measures for selecting the appropriate number of clusters $(k)$ : silhouette analysis and gap analysis. Figure 4 shows the chosen $k$ for each behavioral period and the matching gap curve. In accordance with the aforementioned observations, dividing the neuronal responses during late adaptation to four and five different clusters continued to improve the separation significantly (Fig. $4 c$ ), as tested by comparing with surrogate data, randomly generated from a uniform distribution over the same range of values (see Materials and Methods). In all other periods, preadaptation, early adaptation, and postadaptation, using more than two to three clusters did not improve the separation significantly.

The same procedure was used for all other cases, and we clustered cells in each case according to the chosen $k$. Figures 5 and 6 show the shapes of the clusters for each case. Single-unit responses in MI during preparation for movement $(n=$ 621, 362 from monkey 1 and 259 from monkey 2) (Fig. 5al-a4) consisted of three major clusters. This was the case in the preadaptation period (Fig. 5al) (see also Fig. $3 a$ ) as well as in early adaptation (Fig. 5a2) and postadaptation (Fig. 5a4). Furthermore, the distribution of cells between the three clusters was similar in all these periods $\left(\chi^{2}, p=0.2\right)$. In contrast and as aforementioned, during late adaptation (Fig. 5a3) (see also Fig. 3b,c), patterns of preparatory activity were more diverse and segregated into more clusters. For movement-related activity $(n=598,359$ from monkey 1 and 239 from monkey 2) (Fig. 5b1-b4), MI exhibited the same pattern of responses during all periods, and the distribution of cells between clusters was also similar in all four periods $\left(\chi^{2}, p=0.65\right)$. Note that MRA reflects best the motor command that initializes and generates the movement, before any sensory feedback kicks in, and the finding therefore suggests that it remained the same. However, more clusters were observed in MI in early adaptation when analyzing neuronal responses further into the movement $(+300$ to $+1000 \mathrm{~ms}$; data not shown), reflecting the changes in movement kinematics that occur during adaptation.

A very different behavior was observed for SMA cells (Fig. 6): a complex pattern of activity was observed during early adapta- 
tion in both preparatory activity $(n=149$ from monkey 1) (Fig. 6a2) and movement-related activity $(n=146$ from monkey 1) (Fig. 6b2). In all other periods, the number of clusters was smaller (three), the response patterns remained quite similar and typical, and the distribution of cells between clusters was also $\operatorname{similar}\left(\chi^{2}, p=0.45\right.$ for PA and $p=0.43$ for MRA).

The clustering results are summarized in Figure $7 a$, showing the average number of clusters that best explained the data for SMA and MI and during all periods. A higher number of clusters was observed in SMA during early adaptation for both preparatory activity and movement-related activity, but, as adaptation progresses, a higher number of clusters was observed in MI only for preparatory activity.

\section{Cluster membership of individual cells and response patterns of the clusters}

All shown clusters had a significant modulation (Mann-Whitney $U$ test, comparing activity in the $500 \mathrm{~ms}$ postevent with the $500 \mathrm{~ms}$ preevent, $p<0.05$ ). We also tested each cell individually for a significant modulation in activity (paired $t$ test over single trials, $p<0.05$ ) and compared the number of cells with significant modulation in each cluster separately with that expected from chance (Fisher's exact test, $p<$ 0.05 ); Figure $7 b$ shows that most clusters had a significantly high number of cells with significant modulation.

We then explored the cluster membership of single cells in MI in different periods. To do so, we constructed $N \times M$ matrices to compare between a given behavioral period with the preadaptation period. In such matrix, $N$ and $M$ are the number of clusters in the two periods, respectively, and each entry holds the number of cells that were assigned to cluster $n$ during one period and to cluster $m$ during the other. Thus, we could observe both stability of membership across periods with similar clustering and shifts in membership during adaptation. Figure 8 shows the log ratio of the observed-to-expected probabilities grayscale coded in the main three matrices $(a-c)$, the response patterns of the matching clusters, and the actual number of cells in the right vertical bar graph. For MRA in MI during postadaptation versus preadaptation (Fig. 8a) and for PA in MI (Fig. 8b), the matrices had a clear positive main diagonal, which means that cells tended to keep their cluster membership (see matching response patterns as well); similar results were obtained for other pairs of periods with similar number of clusters. For PA in MI during late adaptation versus preadaptation (Fig. $8 c$ ), it seems that the phasic cluster separated into three clusters with different peaks (bright top three right entries), and some cells became positively active from a previous decreasing cluster; however, these are qualitative observations. All matrices were significantly different from homogenous ( $\chi^{2}$ test, $p<0.01$ for all).

Finally, to quantify the similarity between the response patterns in different behavioral periods, we calculated pairwise correlations (Pearson's correlation coefficient) between the shapes of the clusters. For each combination of two behavioral periods, Figure 9 depicts the average of correlations between the shapes of the clusters in the two periods. This similarity matrix (Fig. 9) quantifies and confirms the aforementioned observation: the response patterns were more similar across conditions that had similar number of clusters and less similar to the patterns seen when the number of clusters was higher.

\section{Discussion}

This study adapted unsupervised clustering for analysis of neuronal responses, allowing for a "natural" classification of evoked responses of single-unit activity. We found that the response patterns in MI and SMA show typical three response patterns (three clusters) during performance of standard, well trained center-out task; however, during adaptation to visuomotor rotations, the response patterns become more variable, and more clusters are needed to represent the different groups. Together with the assumption that cells with similar response patterns (i.e., cells in one cluster) are functionally closer to each other, these findings are consistent with the notion that clusters of cells represent functional groups and that the functional groups are dynamic in nature. Specifically, our results suggest that dynamic reorganization of functional groups takes place during adaptation and that the reorganization is distinct and different in the two cortical areas. In the SMA, the number of clusters increases only during the early stage of adaptation, suggesting that SMA mediates this stage; however, as adaptation proceeds, an increased number of clusters is observed in MI. This reinforces the notion that skill proficiency relies on a representation within low levels of processing (Karni et al., 1998), a suggestion that brings together ideas from the motor and sensory systems (Paz et al., 2004). Furthermore, the changes in MI occurred during preparation for movement rather than during its execution, reflecting the idea that well trained behaviors can be prepared in advance.

What could be the functional role of the increased number of clusters? In our experiment, the animals were overtrained in the standard task, before and after adaptation, in which the direction of the hand is coupled with that of the visual target. The adaptation to visuomotor rotations required dissociation between these 
Pre

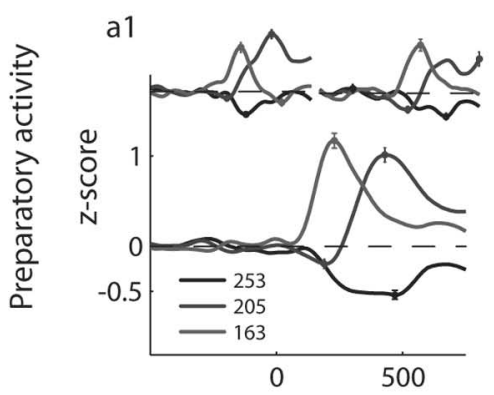

Early

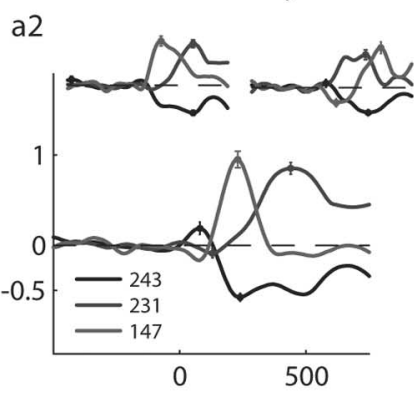

Late

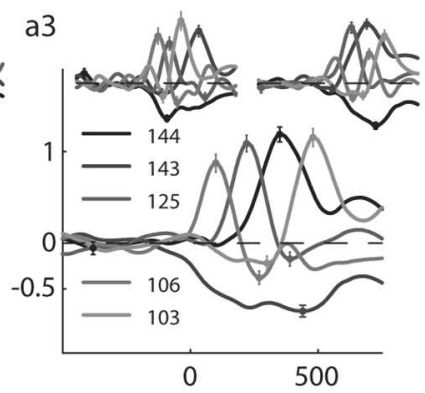

Post

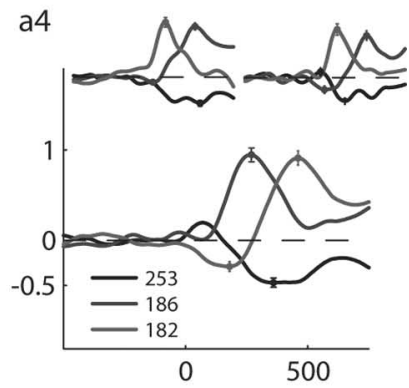

Time from target appearence (ms)
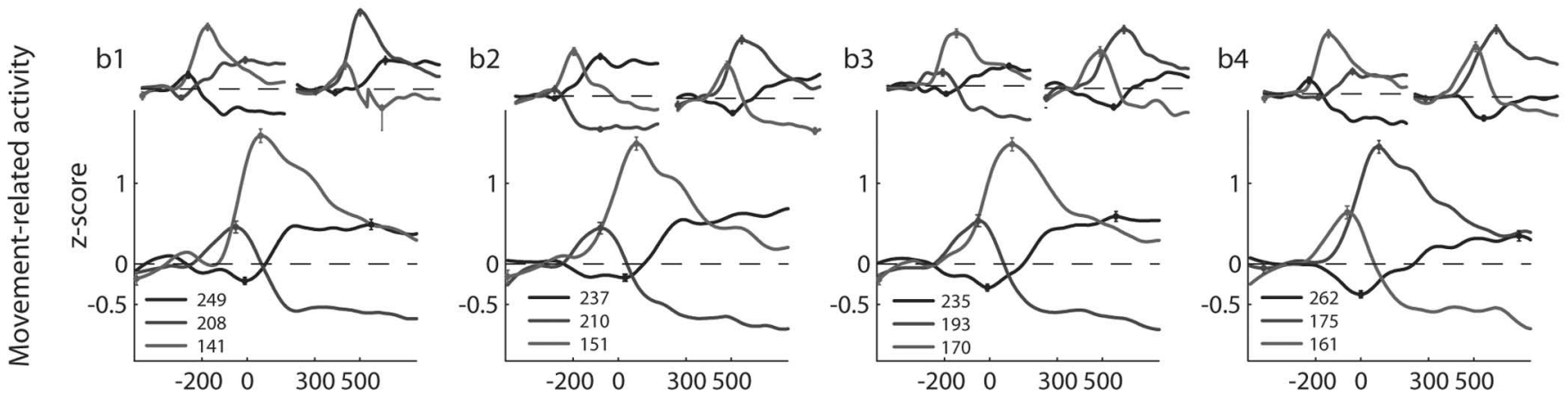

Time from movement onset (ms)

Figure 5. Response patterns in MI. Averaged PETHs from all neurons associated with a cluster, for all clusters. Columns show (from left to right) responses during performance of a standard task before adaptation (preadaptation $\boldsymbol{a} \mathbf{1}, \boldsymbol{b} \mathbf{1})$, early adaptation to visuomotor rotational task $(\boldsymbol{a} \mathbf{2}, \boldsymbol{b} \mathbf{2})$, late adaptation to the visuomotor task $(\boldsymbol{a} \mathbf{3}, \boldsymbol{b} \mathbf{3})$, and performance of standard task after adaptation (postadaptation, $\boldsymbol{a} \mathbf{4}, \boldsymbol{b} \mathbf{4})$. Rows show responses for preparatory activity $(\boldsymbol{a} \mathbf{1}-\boldsymbol{a} \mathbf{4})$ and movement-related activity $(\boldsymbol{b} \mathbf{1}-\boldsymbol{b} \mathbf{4})$. For presentation purposes, the PETHs are shown in a time window from $-500 \mathrm{to}+750 \mathrm{~ms}$ around a behavioral event: target appearance (top row, preparatory activity) and movement onset (bottom row, movement-related activity). The clustering itself was performed using a smaller window of $500 \mathrm{~ms}$ from 0 to $500 \mathrm{~ms}$ around the target appearance and from -200 to $+300 \mathrm{~ms}$ around the movement onset. Clusters are sorted according to the number of neurons that are associated with them and presented in a matching grayscale. Insets show the clusters when analyzing separately neurons from the first monkey (left) and from the second (right). Note that typical response patterns with a small number of clusters (3) were observed in most cases, but a higher number (5) was observed for preparatory activity during late adaptation (a3).

Pre

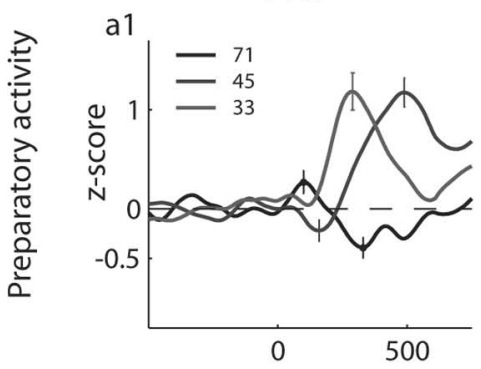

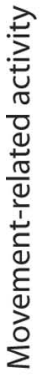

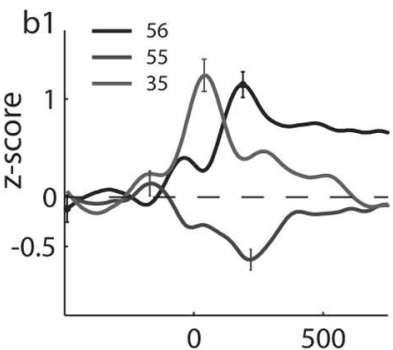

Early

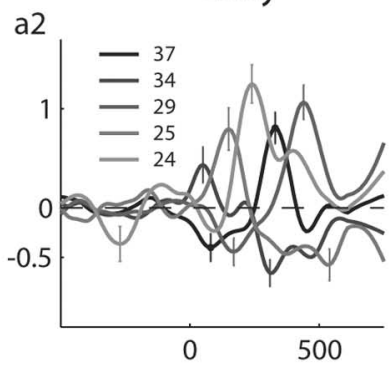

Late

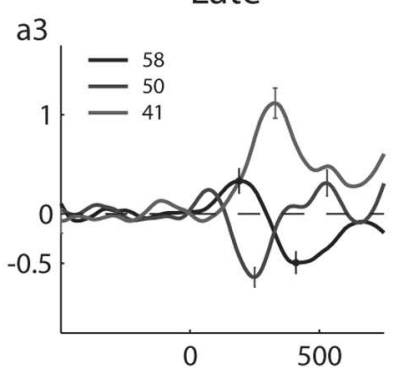

Time from target appearence (ms)

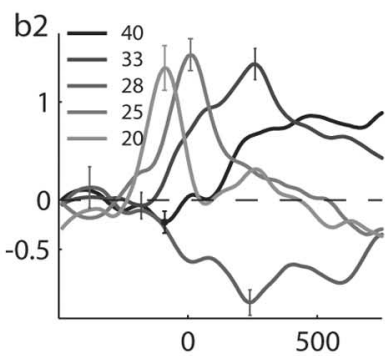

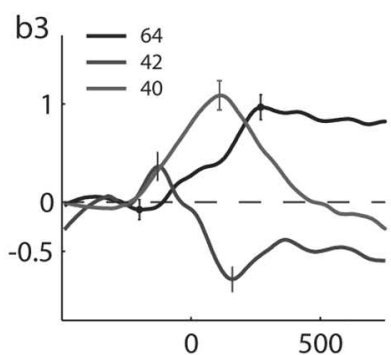

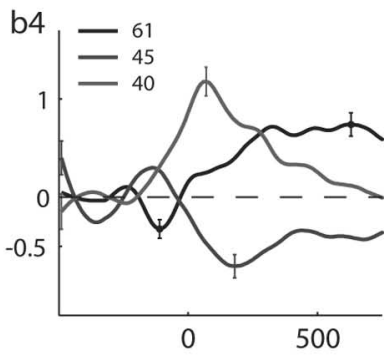

Time from movement onset (ms)

Figure 6. Response patterns in SMA. Same format as in Figure 5. For SMA, typical response patterns with a small number of clusters (3) were observed in most cases, but a higher number (5) was observed for preparatory activity and for movement-related activity during early adaptation $(\mathbf{a} \mathbf{2}, \boldsymbol{b} \mathbf{2})$. 
two directions and therefore the learning of a new kinematics-to-dynamics transformation (the transformation from a desired target location in visual coordinates to a desired hand trajectory and then to the required muscle activations). This could be performed by several mechanisms (Lurito et al., 1991; Shen and Alexander, 1997a; Cisek and Scott, 1999; Padoa-Schioppa et al., 2002), all demanding recruitment of different populations of cells at different times during the preparation for and/or execution of movement. In accordance, during adaptation, we found higher variability of the time-topeak activation, and the multiple clusters that emerged during adaptation differed mainly in the time of their peak. Thus, it is possible that the different clusters reflect different stages of computation and processing within the cortical network.

A recent study of force-field adaptation observed gradual and continuous shifts in preferred direction of cells in the SMA during their preparatory activity and concluded that it reflects the movement dynamics gradually, starting from a kinematics-related signal (Padoa-Schioppa et al., 2002). If the underlying transformation is a continuous computation, then the actual number of clusters reported here is arbitrary and only reflects the increased variability of peak times rather than specific separate subpopulations with clear distinctive functions. Alternatively, we recently suggested that a directional-specific elevation of activity observed in MI can serve to "win over" the default natural behavior and produce the correct movement under the visuomotor transformation (Paz et al., 2003). This result, together with a more rigid view of the number of clusters reported here, is more in line with the "response substitution" view (Cisek and Scott, 1999), in which the default input to motor areas is in the direction of the visual target (perhaps because it is the natural and the well trained response) but is then aborted and replaced by inputs that signify the newly learned direction. Differences in the two underlying mechanisms could also result from differences in dynamic versus kinematic adaptations.

The changes in MI were observed at late stages of adaptation when the directional errors were reduced almost to the plateau level and adaptation seems to be complete. This is in agreement with previous studies that have examined learning on a longer time scale (i.e., across sessions and days) (Karni et al., 1998; Cohen and Nicolelis, 2004; Kleim et al., 2004). There are several possibilities that could explain the similar finding even within a single session. First, because the visuomotor task in-
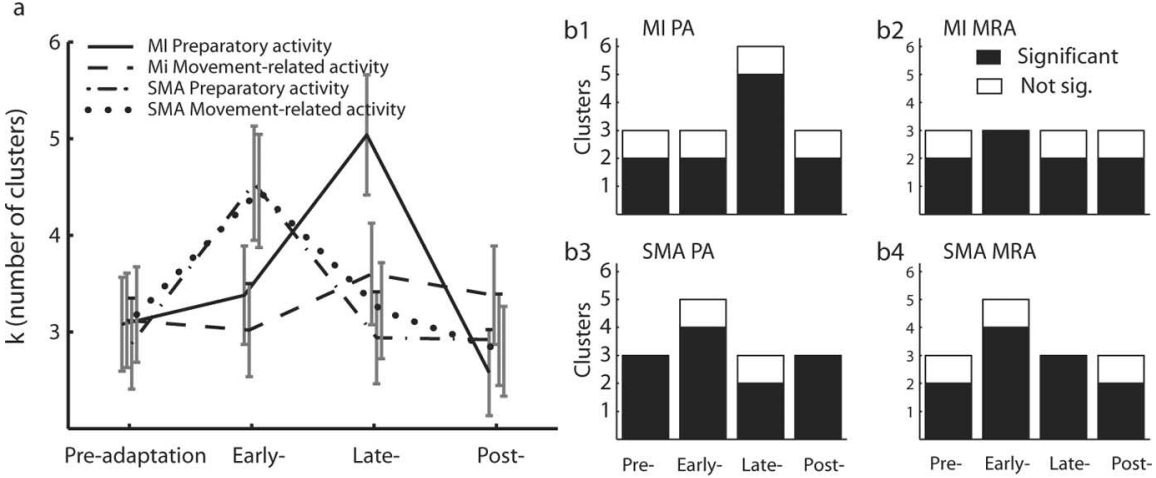

Figure 7. Number of clusters best explaining the neuronal responses in SMA and MI. $\boldsymbol{a}$, The average number of clusters for all cases. The gray vertical bars show 0.05 confidence intervals calculated by silhouette analysis based on fitting a Poisson distribution to bootstrap statistics. A significant increase in the number of clusters was observed in MI for preparatory activity during late adaptation and in SMA during early adaptation for both preparatory activity and movement-related activity. $\boldsymbol{b}$, Filled rectangles represent clusters with a significant number of cells with significant modulation. Each cell was tested individually for a significant modulation of activity (paired $t$ test over single trials, $p<0.05$ ), and the number of such cells within each cluster separately was tested against the chance ratio of $5 \%$ (Fisher's exact test, $p<0.05$ ). $\boldsymbol{b} 1$, PA in Ml; $\boldsymbol{b 2}$, MRA in Ml; $\boldsymbol{b} 3$, PA in SMA; $\boldsymbol{b} 4$, MRA in SMA.
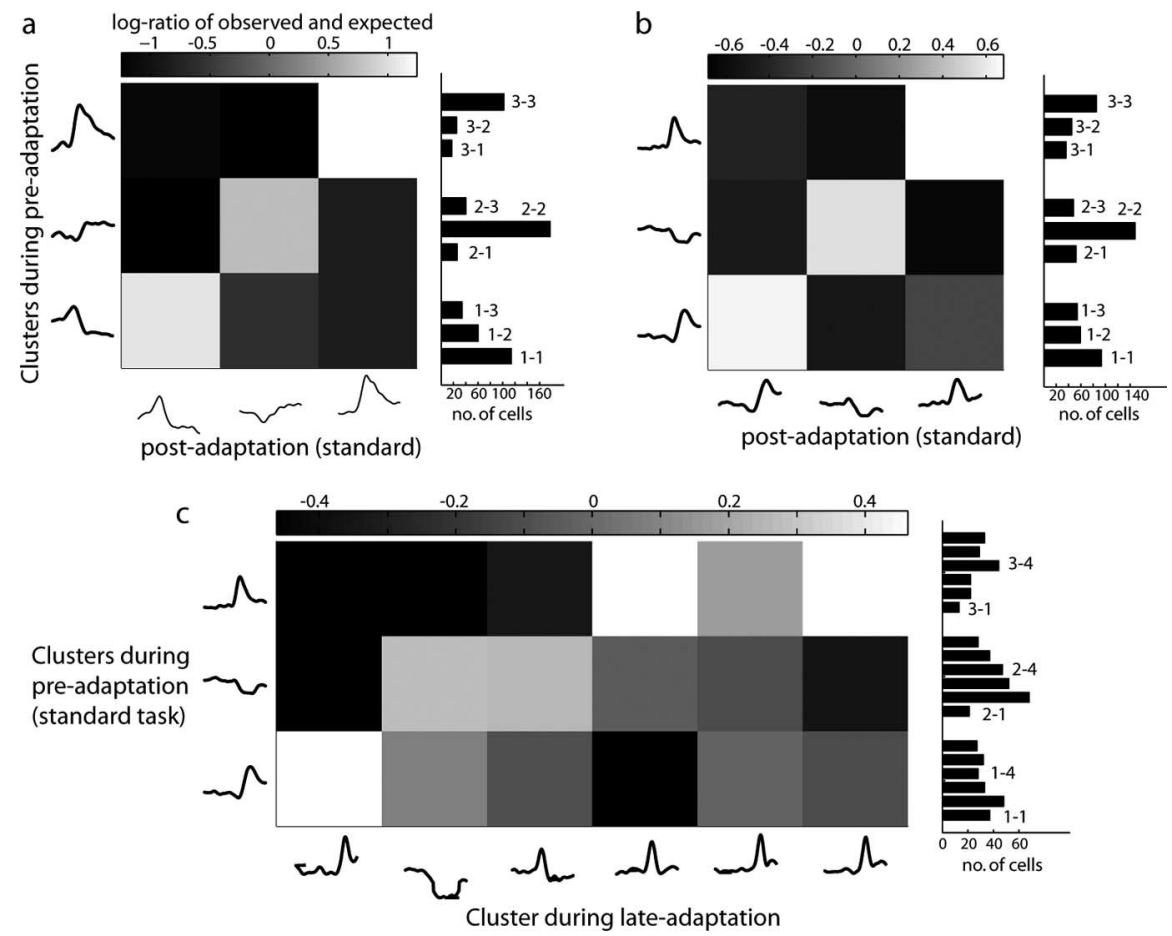

Figure 8. Changes in cluster membership of individual neurons. Each entry in a matrix designates a combination of a cluster from a given behavioral period (rows) to the preadaptation period and represents the number of cells that were assigned to these clusters in the two behavioral periods accordingly. The matrix is then normalized by its sum to obtain joint probabilities and divided by the product distribution obtained from multiplying the marginal distributions; the log of this ratio

$$
\log \left(\frac{p(x, y)}{p(x) \times p(y)}\right)
$$

is presented by grayscale. Thus, a positive number represents a higher than expected number of cells for this entry and a negative number vice versa. The response patterns of the clusters are shown to the sides, and the actual number of cells in each entry is shown by the bar graph to the right. $\boldsymbol{a}$, MRA in MI during preadaptation versus postadaptation. A clear diagonal means that cells tend to keep their cluster membership (see the matching response patterns). $\boldsymbol{b}$, PA in MI during preadaptation versus postadaptation. c, PA in Ml during prelearning versus late adaptation.

duced visual directional errors, the monkeys might have used a strategy that minimizes errors in this relevant dimension (Todorov, 2004), but improvement of other less relevant movement parameters emerges naturally and occurs more slowly. Sec- 
Preparatory activity
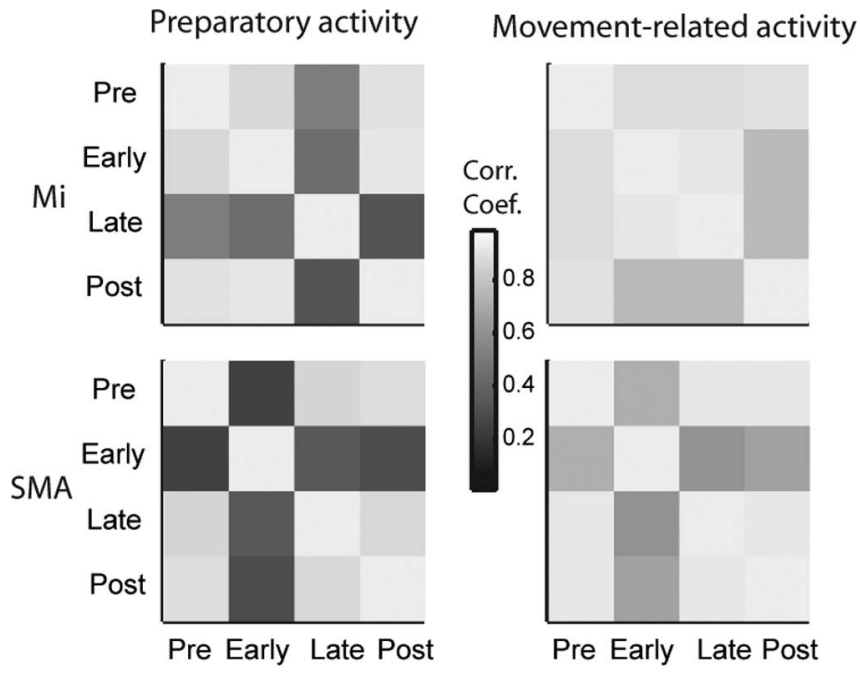

Figure 9. Similarities of response patterns. Similarity (symmetric) matrices between response patterns of clusters in the four behavioral periods (preadaptation, early adaptation, late adaptation, and postadaptation). Each value is the average Pearson's correlation coefficient (average over correlations between the shapes of the clusters in the two periods). For preparatory activity in $\mathrm{MI}$ (top left), the matrix shows that the patterns during the late adaptation phase were the most dissimilar to others (darker strips). For movement-related activity in $\mathrm{MI}$ (top right), high correlations ( $>0.6$ ) were observed between all periods. The preparatory activity in SMA (bottom left) in early adaptation was the most dissimilar to all other periods. Movement-related activity in SMA (bottom right) during early adaptation also differs from all other periods.

ond, it is likely that dynamic changes that generate the same trajectory more efficiently follow the kinematic changes that we observed. For example, it has been demonstrated that high viscoelastic forces are used to stabilize and generate the correct movement at early stages of adaptation but decrease when a new internal model for the learned skill is formed (Osu et al., 2002; Paz et al., 2003). In accordance with these suggestions, the error variance was still improving during late adaptation (Fig. 1e). Finally, the late activation in MI could reflect the shift of the newly acquired skill from working memory to a

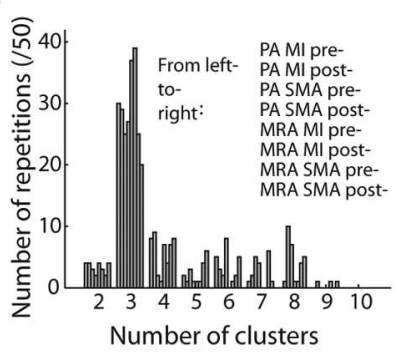

b

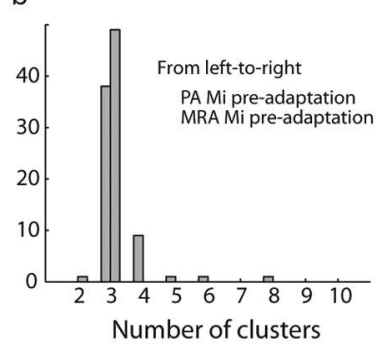

long-term memory (Shadmehr and Holcomb, 1997; Muellbacher et al., 2002).

This late involvement of a primary area could account for the locality of adaptation in our task. The monkeys were trained for only one target in space and showed poor generalization to other directions (Paz et al., 2003, 2005), as observed previously in humans (Krakauer et al., 2000). This is consistent with the hypothesis that learning starts at areas "higher in the hierarchy" (SMA in the present case) and only later forms in primary areas (MI). Because the representation in a primary area is less abstract, then as learning progresses, transfer (generalization) reduces and specificity (locality) increases (Hochstein and Ahissar, 2002; Korman et al., 2003). In the current paradigm, it is possible that the spatial specificity is determined by the shape of the primitives of representation (Donchin et al., 2003), namely, directional tuning curves of MI cells (Georgopoulos et al., 1982), and adaptation is therefore directionally confined. Indeed, the width of neuronal tuning curves of MI cells, the improvement in the information content conveyed by the neurons, and the width of the behavioral generalization function match quite closely ( $\mathrm{Paz}$ and Vaadia, 2004).

What is the relationship between the adaptation-dependant changes in SMA and MI? One intriguing possibility is that SMA instructs MI during adaptation; another is that SMA merely generates the new complex movement, whereas MI either "learns" by itself or is instructed by other areas. Supporting both hypotheses, Aizawa et al. (1991) found that activity in SMA is less pronounced for well trained motor tasks but becomes more abundant after lesions to MI. Unfortunately, we cannot dissociate between the two hypotheses as well, because the dual role of SMA as a premotor area and as an area intimately related to the generation of movement is in line with both suggestions. Other premotor areas that we did not examine could also take part in the process: the presupplementary SMA (preSMA), the dorsal premotor area, and the ventral premotor area all code for the visual aspects of the task, complex aspects of the motor behavior, and learning of complex skills (Alexander and Crutcher, 1990; Mitz et al., 1991; Matsuzaka et al., 1992; Matsuzaka and Tanji, 1996; Shen and Alexander, 1997b; Brasted and Wise, 2004). Only one study has examined brain activations during adaptation to rotational transformations (Krakauer et al., 2004).

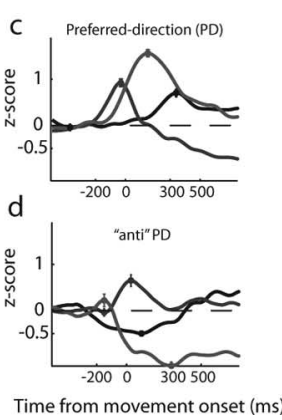

Time from movement onset (ms)
Figure 10. The number of clusters that best describes the data are robust to the number of trials and sensitivity of the cells to direction. Distributions of $k$ values for 50 repetitions of random samplings with replacement from neuronal data during preadaptation and postadaptation periods. For each repetition, we calculated the $k$ value (number of clusters best describing the data) using silhouette analysis. All distributions are clearly centered on three clusters, showing that the increased number of clusters found during adaptation does not result from a larger number of trials that was used for the standard task ( $\boldsymbol{a}$, based on samplings of 10 trials from preadaptation and postadaptation periods in both areas and activity periods) or from pooling the eight directions used during the standard task $[\boldsymbol{b}$, based on analysis of preadaptation in Ml using only movements to the preferred direction of the cells and the two adjacent directions $\left.\left( \pm 45^{\circ}\right)\right]$. $\boldsymbol{c}, \boldsymbol{d}$, Response patterns for MRA in MI during the preadaptation period using only movements to the preferred direction of the cells $(\boldsymbol{c})$ and the direction opposite to it $(\boldsymbol{d})$. Whereas slight and expected differences can be observed, the responses of the clusters show similar temporal patterns to those found using all movement directions pooled together.
This study used imaging (positron emission tomography) techniques and found activations in preSMA during the rapid phase of adaptation but did not observe any difference in activation in the SMA proper during that phase. However, our analysis focused on changes in diversity of patterns and subpopulations of neurons over a few hundreds of milliseconds; these changes could be low-pass filtered by the imaging technique and remain undetected. Finally, it is important to note that this study attempts to detect changes in cortical processing using one type of analysis. Other analyses methods might reveal additional changes, and we cannot preclude the possibility that MI also participates in early stages of adaptation (Cohen and Nicolelis, 2004) and that SMA may also contribute to later stages (PadoaSchioppa et al., 2002).

Our interpretations rely on differences 
that occur when the monkey switched from performance of a standard task to the adaptation task. One could argue that the higher number of clusters observed is not related to adaptation but to other task differences: the higher number of trials and the eight directions used in the standard task. However, this is highly unlikely. First, there were cases of adaptation-dependant activity that did not result in a higher number of clusters (Figs. 5, MI during early phases, and 6, SMA during late phases). Second, we tested and found that the increase in number of clusters was only slightly, if at all, sensitive to the number of trials (Fig. 10a) and to the eight different directions (Fig. 10b-d).

To conclude, we suggest that the clustering exposed subpopulations that emerge at different stages and levels of the computation in the neural network during adaptation to visuomotor rotations. In our study, these subpopulations first emerge in the supplementary motor area and only later in the primary motor area. Future studies should expand and focus on identifying functionally different roles for the different classes of cells.

\section{References}

Aizawa H, Inase M, Mushiake H, Shima K, Tanji J (1991) Reorganization of activity in the supplementary motor area associated with motor learning and functional recovery. Exp Brain Res 84:668-671.

Alexander GE, Crutcher MD (1990) Preparation for movement: neural representations of intended direction in three motor areas of the monkey. J Neurophysiol 64:133-150.

Brasted PJ, Wise SP (2004) Comparison of learning-related neuronal activity in the dorsal premotor cortex and striatum. Eur J Neurosci 19:721-740.

Cisek P, Scott SH (1999) An alternative interpretation of population vector rotation in macaque motor cortex. Neurosci Lett 272:1-4.

Cohen D, Nicolelis MA (2004) Reduction of single-neuron firing uncertainty by cortical ensembles during motor skill learning. J Neurosci 24:3574-3582.

Crammond DJ, Kalaska JF (2000) Prior information in motor and premotor cortex: activity during the delay period and effect on pre-movement activity. J Neurophysiol 84:986-1005.

Crutcher MD, Alexander GE (1990) Movement-related neuronal activity selectively coding either direction or muscle pattern in three motor areas of the monkey. J Neurophysiol 64:151-163.

Donchin O, Francis JT, Shadmehr R (2003) Quantifying generalization from trial-by-trial behavior of adaptive systems that learn with basis functions: theory and experiments in human motor control. J Neurosci 23:9032-9045.

Doyon J, Gaudreau D, Laforce Jr R, Castonguay M, Bedard PJ, Bedard F, Bouchard JP (1997) Role of the striatum, cerebellum, and frontal lobes in the learning of a visuomotor sequence. Brain Cogn 34:218-245.

Fellous JM, Tiesinga PH, Thomas PJ, Sejnowski TJ (2004) Discovering spike patterns in neuronal responses. J Neurosci 24:2989-3001.

Georgopoulos AP, Kalaska JF, Caminiti R, Massey JT (1982) On the relations between the direction of two-dimensional arm movements and cell discharge in primate motor cortex. J Neurosci 2:1527-1537.

Hartigan JA (1975) Clustering algorithms. New York: Wiley.

He S-Q, Dum RP, Strick PL (1993) Topographic organization of corticospinal projections from the frontal lobe: motor areas on the lateral surface of the hemisphere. J Neurosci 13:952-980.

He SQ, Dum RP, Strick PL (1995) Topographic organization of corticospinal projections from the frontal lobe: motor areas on the medial surface of the hemisphere. J Neurosci 15:3284-3306.

Hikosaka O, Nakamura K, Sakai K, Nakahara H (2002) Central mechanisms of motor skill learning. Curr Opin Neurobiol 12:217-222.

Hochstein S, Ahissar M (2002) View from the top: hierarchies and reverse hierarchies in the visual system. Neuron 36:791-804.

Karni A, Meyer G, Rey HC, Jezzard P, Adams MM, Turner R, Ungerleider LG (1998) The acquisition of skilled motor performance: fast and slow experience-driven changes in primary motor cortex. Proc Natl Acad Sci USA 95:861-868.
Kaufman L, Rousseeuw PJ (1990) Finding groups in data: an introduction to cluster analysis. New York: Wiley.

Kleim JA, Hogg TM, VandenBerg PM, Cooper NR, Bruneau R, Remple M (2004) Cortical synaptogenesis and motor map reorganization occur during late, but not early, phase of motor skill learning. J Neurosci 24:628-633.

Korman M, Raz N, Flash T, Karni A (2003) Multiple shifts in the representation of a motor sequence during the acquisition of skilled performance. Proc Natl Acad Sci USA 100:12492-12497.

Krakauer JW, Pine ZM, Ghilardi MF, Ghez C (2000) Learning of visuomotor transformations for vectorial planning of reaching trajectories. J Neurosci 20:8916-8924.

Krakauer JW, Ghilardi MF, Mentis M, Barnes A, Veytsman M, Eidelberg D, Ghez C (2004) Differential cortical and subcortical activations in learning rotations and gains for reaching: a PET study. J Neurophysiol 91:924-933.

Laubach M, Wessberg J, Nicolelis MA (2000) Cortical ensemble activity increasingly predicts behaviour outcomes during learning of a motor task. Nature 405:567-571.

Lee D, Quessy S (2003) Activity in the supplementary motor area related to learning and performance during a sequential visuomotor task. J Neurophysiol 89:1039-1056.

Li CS, Padoa-Schioppa C, Bizzi E (2001) Neuronal correlates of motor performance and motor learning in the primary motor cortex of monkeys adapting to an external force field. Neuron 30:593-607.

Luppino G, Matelli M, Camarda R, Rizzolatti G (1993) Corticocortical connections of area F3 (SMA-proper) and area F6 (pre-SMA) in the macaque monkey. J Comp Neurol 338:114-140.

Lurito JT, Georgakopoulos T, Georgopoulos AP (1991) Cognitive spatialmotor processes. 7. The making of movements at an angle from a stimulus direction: studies of motor cortical activity at the single cell and population levels. Exp Brain Res 87:562-580.

Matsuzaka Y, Tanji J (1996) Changing directions of forthcoming arm movements: neuronal activity in the presupplementary and supplementary motor area of monkey cerebral cortex. J Neurophysiol 76:2327-2342.

Matsuzaka Y, Aizawa H, Tanji J (1992) A motor area rostral to the supplementary motor area (presupplementary motor area) in the monkey: neuronal activity during a learned motor task. J Neurophysiol 68:653-662.

Mitz AR, Godschalk M, Wise SP (1991) Learning-dependent neuronal activity in the premotor cortex: activity during the acquisition of conditional motor associations. J Neurosci 11:1855-1872.

Muellbacher W, Ziemann U, Wissel J, Dang N, Kofler M, Facchini S, Boroojerdi B, Poewe W, Hallett M (2002) Early consolidation in human primary motor cortex. Nature 415:640-644.

Nakamura K, Sakai K, Hikosaka O (1998) Neuronal activity in medial frontal cortex during learning of sequential procedures. J Neurophysiol 80:2671-2687.

Ng A, Jordan M, Weiss Y (2001) On spectral clustering: analysis and an algorithm. Adv Neural Inform Proc Systems 14:849-856.

Osu R, Franklin DW, Kato H, Gomi H, Domen K, Yoshioka T, Kawato M (2002) Short- and long-term changes in joint co-contraction associated with motor learning as revealed from surface EMG. J Neurophysiol 88:991-1004.

Padoa-Schioppa C, Li CS-R, Bizzi E (2002) Neuronal correlates of kinematics-to-dynamics transformation in the supplementary motor area. Neuron 36:751-765.

Padoa-Schioppa C, Li CS, Bizzi E (2004) Neuronal activity in the supplementary motor area of monkeys adapting to a new dynamic environment. J Neurophysiol 91:449-473.

Paz R, Vaadia E (2004) Learning-induced improvement in encoding and decoding of specific movement directions by neurons in the primary motor cortex. PLoS Biol 2:E45.

PazR, Boraud T, Natan C, Bergman H, Vaadia E (2003) Preparatory activity in motor cortex reflects learning of local visuomotor skills. Nat Neurosci 6:882-890.

Paz R, Wise SP, Vaadia E (2004) Viewing and doing: similar cortical mechanisms for perceptual and motor learning. Trends Neurosci 27:496-503. 
Paz R, Nathan C, Boraud T, Bergman H, Vaadia E (2005) Acquisition and generalization of visuomotor transformations by nonhuman primates. Exp Brain Res 161:209-219.

Porter R, Lemon RN (1993) Corticospinal function and voluntary movement. New York: Oxford UP.

Rieke F, Warland D, De Ruyter van Steveninck RR, Bialek W (1997) Spikes: exploring the neural code. Cambridge: MIT.

Sanes JN, Donoghue JP (2000) Plasticity and primary motor cortex. Annu Rev Neurosci 23:393-415.

Schreiber S, Fellous JM, Tiesinga PH, Sejnowski TJ (2003) A new correlationbased measure of spike timing reliability. Neurocomputing 52-54:925-931.

Scott SH (2004) Optimal feedback control and the neural basis of volitional motor control. Nat Rev Neurosci 5:532-546.

Shadmehr R, Holcomb HH (1997) Neural correlates of motor memory consolidation. Science 277:821-825.
Shen L, Alexander GE (1997a) Neural correlates of a spatial sensory-tomotor transformation in primary motor cortex. J Neurophysiol 77:1171-1194.

Shen L, Alexander GE (1997b) Preferential representation of instructed target location versus limb trajectory in dorsal premotor area. J Neurophysiol 77:1195-1212.

Tanji J (1994) The supplementary motor area in the cerebral cortex. Neurosci Res 19:251-268.

Tibshirani R, Walther G, Hastie T (2001) Estimating the number of clusters in a dataset via the gap statistic. J R Stat Soc 32:411-423.

Todorov E (2004) Optimality principles in sensorimotor control. Nat Neurosci 7:907-915.

Ungerleider LG, Doyon J, Karni A (2002) Imaging brain plasticity during motor skill learning. Neurobiol Learn Mem 78:553-564. 